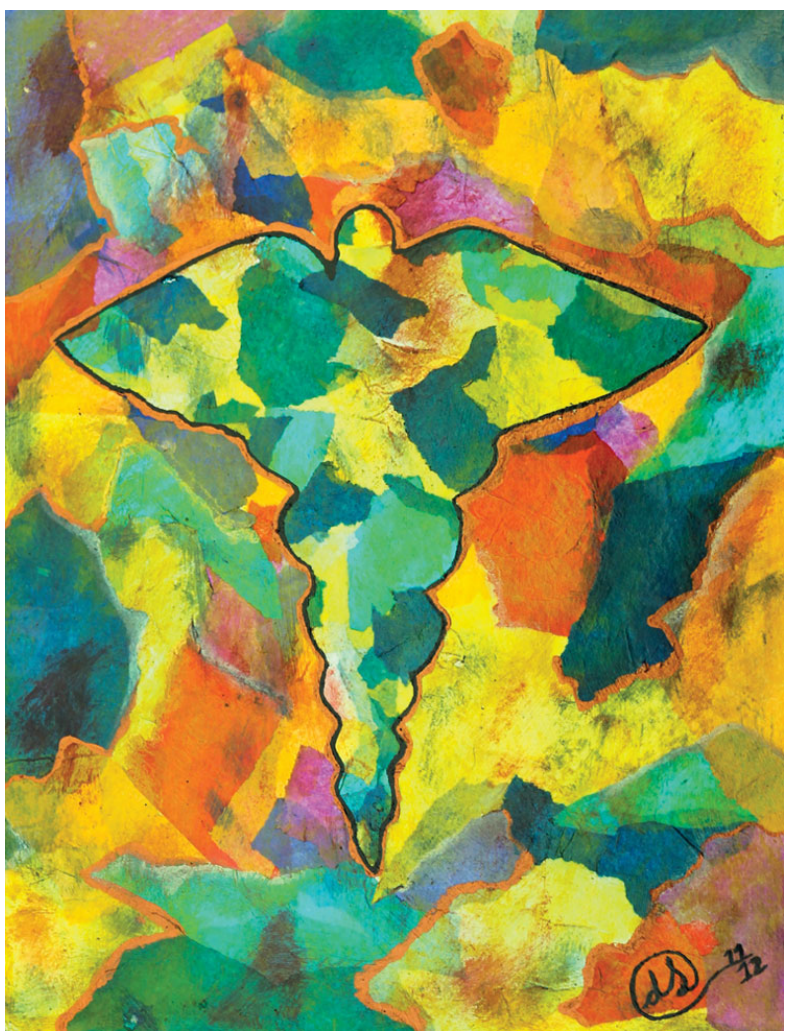

\title{
The color of medicine
}

\author{
Diana Salinas
}

College of Human Medicine, Michigan State University

I will not permit considerations of religion, nationality, race, gender, politics, socioeconomic standing, or sexual orientation to intervene between my duty and my patient.

T

his bullet point from the physician's oath is engraved around the margins to remind us that as physicians we should treat and care for each patient equally and in an unbiased manner, but at the same time understand that a universal treatment plan will not apply to all patients. This requires us as physicians to develop cultural competency and be aware of patient diversity.

The symbol of medicine in the center represents the power granted to us as physicians. However, we have to be careful not to abuse that power. Instead, we have to do our best to meet eye to eye with our patients to encourage communication and develop a shared treatment plan. Therefore, the symbol of medicine stands out, but if we remove the margin, all the colors blend because, at the end of the day, despite our role as physicians, we are still people who can get sick and make mistakes.

Medicine is about teamwork. We bring our own unique experiences to the field, and we need to work together regardless of our title or specialty choice. This includes other healthcare professionals and support staff. To represent this, the symbol of medicine is composed of different colors to showcase the variety of careers that encompass the field of medicine. 